\title{
Repeatability of Optic Nerve Head Parameters Measured by Spectral-Domain OCT in Healthy Eyes
}

Giacomo Savini, MD; Michele Carbonelli, MD; Vincenzo Parisi, MD; Piero Barboni, MD

- BACKGROUND AND OBJECTIVE: To evaluate the repeatability of optic nerve head $(\mathrm{ONH})$ measurements by spectral-domain optical coherence tomography.

- PATIENTS AND METHODS: Three scans were acquired in 32 healthy subjects during one session. Using Cirrus HD-OCT (Carl Zeiss Meditec, Dublin, CA), the cup-to-disc ratio (CDR), disc area, rim area, cup volume, and horizontal and vertical CDRs were investigated. Repeatability was assessed by the coefficient of variation $(\mathrm{COV})$, the test-retest intrasession variability, and the intraclass correlation coefficient (ICC).

\section{INTRODUCTION}

Optical coherence tomography (OCT) is a noninvasive imaging technique for creating in vivo crosssectional images of biological tissue. ${ }^{1}$ Detailed structural images result, for which quantitative analyses have been developed to support clinical interpretation. Time-domain OCT (TD-OCT) using Stratus OCT
- RESULTS: Good repeatability was achieved for all parameters, with a COV of $4.23 \%$ or less and ICCs of 0.98 or greater for all measurements. Test-retest intrasession variability was 0.024 for CDR, $0.121 \mathrm{~mm}^{2}$ for disc area, $0.087 \mathrm{~mm}^{2}$ for rim area, $0.017 \mathrm{~mm}^{3}$ for cup volume, and 0.048 for horizontal and vertical CDR.

CONCLUSION: In healthy eyes, Cirrus HD-OCT provides repeatable measurements of $\mathrm{ONH}$ parameters.

[Ophthalmic Surg Lasers Imaging 2011;42:209215.]

(Carl Zeiss Meditec, Dublin, CA) is well established in clinical practice, but the emphasis is now shifting to the more recent spectral-domain OCT (SD-OCT) devices, which have faster acquisition rates and higher axial resolutions. ${ }^{2,3}$ The increase in the speed of the SD-OCT devices has facilitated the collection of more data, resulting in higher sampling densities and true volumetric imaging.

From G.B. Bietti Eye Foundation-IRCCS (GS, MC, VP), Rome; Studio Oculistico d'Azeglio (PB), Bologna; and the Department of Neurological Sciences (PB), University of Bologna, Bologna, Italy.

Originally submitted October 22, 2010. Accepted for publication February 4, 2011. Posted online March 3, 2011.

The authors have no financial or proprietary interest in the materials presented herein.

Address correspondence to Giacomo Savini, MD, G.B. Bietti Eye Foundation-IRCCS, Via Livenza 3, Rome, Italy. E-mail: giacomo.savini@alice.it

doi: 10.3928/15428877-20110224-02 
In the context of optic nerve pathologies, OCT has been used to evaluate the peripapillary retinal nerve fiber layer (RNFL) in many diseases, including glaucoma, nonarteritic anterior ischemic optic neuropathy, optic neuritis, and Leber's hereditary optic neuropathy. ${ }^{4-8}$ In all of these pathologies, OCT has been able to detect and follow changes in RNFL thickness. Recently, SD-OCT has been reported to improve the already good repeatability of conventional TD-OCT in measuring RNFL thickness. ${ }^{9,10}$

OCT can also be used to analyze optic nerve head (ONH) parameters, such as disc area, rim area, or cupto-disc ratio, with good repeatability and reproducibility. ${ }^{11,12}$ This feature has received less attention, although several studies have shown that $\mathrm{ONH}$ analysis improves our ability to discriminate between healthy and glaucomatous eyes. ${ }^{13-16}$ Moreover, ONH parameters have been reported to play an important role in other diseases, such as Leber's hereditary optic neuropathy and dominant optic atrophy. ${ }^{17,18}$ Given the importance of $\mathrm{ONH}$ analysis and the recent development of dedicated software to be used for this purpose with Cirrus HD-OCT (Carl Zeiss Meditec), a commercially available imaging device that uses the SD-OCT technology, we were prompted to investigate the repeatability of its measurements in a sample of healthy eyes.

\section{PATIENTS AND METHODS}

Thirty-three eyes of 33 healthy subjects were prospectively enrolled in a private practice setting for this observational case series. The study population consisted of consecutive patients with minor refractive disorders. All subjects underwent a complete ophthalmologic examination, including visual acuity measurement, intraocular pressure measurement, slit-lamp biomicroscopy, and indirect ophthalmoscopy, to determine eligibility. Inclusion criteria were: best-corrected visual acuity of better than $20 / 25$, refractive error between -3 and +3 diopters of sphere or between -2 and +2 diopters of cylinder, normal intraocular pressure less than $21 \mathrm{~mm} \mathrm{Hg}$, normal appearance of the optic disc, no peripapillary atrophy at fundus examination, no significant ocular disease found by routine ophthalmological examination, and no history of glaucoma in the family and/or systemic diseases with possible ocular involvement, such as diabetes mellitus.

Three individual $200 \times 200$ cube optic disc scans were obtained with Cirrus HD-OCT (software version 5.0) within a single session, without pupil dilation. The subject remained seated between measurements, but was repositioned in the headrest for each measurement. Once the subject was properly aligned, the iris was brought into view and the scanning laser ophthalmoscopic image was focused by adjusting for refractive error. As the optic nerve head was centered on the live scanning laser ophthalmoscopic image using the internal fixation cross, centering (Z-offset) and enhancement (polarization) were optimized and then the scan was acquired. The Optic Disc Cube $200 \times 200$ protocol comprises $200 \mathrm{~B}$-scans; each B-scan comprises 200 A-scans, where an A-scan is the instantaneously acquired reflectivity profile of the back-scattered light acquired by the device. The profile has an axial depth of $2 \mathrm{~mm}$, and is made up of 1,024 samples (pixels). A total of 27,000 A-scans are acquired per second. Each B-scan has a 6-mm horizontal field of view, and the 200 B-scans are stacked over a vertical distance of 6 $\mathrm{mm}$. The resulting volume is therefore a set of $200 \times$ $200 \times 1,024$ data points covering a volume of $6 \times 6$ $\times 2 \mathrm{~mm}$.

The quality of Cirrus HD-OCT scans was assessed by an experienced examiner. To be considered of good quality, the scans had to have focused images from the ocular fundus, a signal strength of at least 6 (on a scale from 1 to 10 ), and no signs of involuntary eye movement during the scan (as evidenced by checking the en-face images for motion). Only scans without overt algorithm failure in detecting the $\mathrm{ONH}$ limits were included in the study. If a scan was classified as unacceptable, the patient was excluded from the study.

The ONH analysis was performed on this data set using 5.0 software. The ONH parameters reported result from a fully automatic algorithm that defines both the disc and cup margins within the three-dimensional data cube. The disc margin is defined as the termination of Bruch's membrane (also referred to as "neural canal opening" or "Bruch's membrane opening"). 19,20 Increased resolution of Cirrus HD-OCT with respect to Stratus OCT (whose transverse resolution is $128 \mathrm{~A}$ scans) can be observed in the figure. The cup margin is defined using a proprietary algorithm that measures the neuroretinal rim as it exits the eye. It is designed to emulate the cup margin, which would be manually defined by a clinician based on an ophthalmic examination. From these landmarks the following param- 


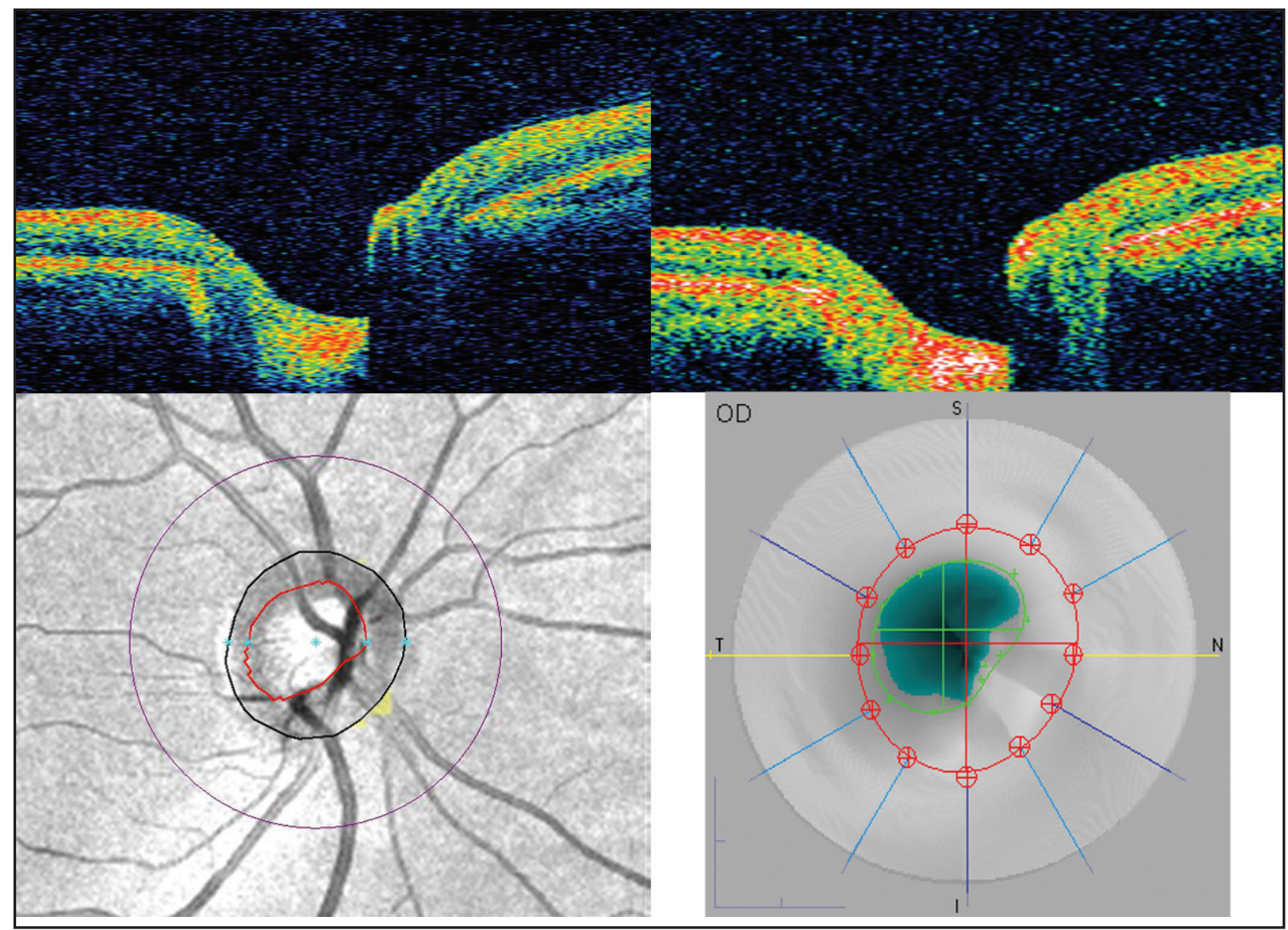

Figure. Optic nerve head imaging by spectral-domain and time-domain optical coherence tomography (OCT). Top: horizontal scan of the same optic nerve head by spectral-domain OCT (left) and time-domain OCT (right). Bottom: optic disc and cup edges reconstructed by spectral-domain OCT (left) and time-domain OCT (right).

eters can be derived and were analyzed in this study: (1) cup-to-disc ratio $(\mathrm{CDR})=$ average $\mathrm{CDR}$, defined as the square root of the cup area divided by the disc area; (2) disc area $=$ the total area within the disc margin $\left(\mathrm{mm}^{2}\right)$; (3) rim area $=$ the area of the neuroretinal rim $\left(\mathrm{mm}^{2}\right) ;(4)$ cup volume $=$ the volume of the $\operatorname{cup}\left(\mathrm{mm}^{3}\right)$; (5) horizontal CDR = the ratio of the cup diameter to the disc diameter in the horizontal meridian; and (6) vertical $\mathrm{CDR}=$ the ratio of the cup diameter to the disc diameter in the vertical meridian.

All participants gave their informed consent according to the Declaration of Helsinki and the study was approved by the internal review board of the University of Bologna Department of Neurological Sciences.

\section{Statistics}

For the purposes of the current study, the term repeatability was used according to the definition of the British
Standards Institute, which considers it a part of accuracy. ${ }^{21}$ Accuracy, as formally defined by BS ISO 5725-1, includes trueness and precision. Trueness is the inverse of bias and is determined by comparing the measurement result with the accepted reference (conventional true) value. Precision is the inverse of statistical uncertainty and is normally expressed in terms of standard deviation (SD). The factors involved include (1) the operator, (2) the equipment used, (3) the equipment calibration, (4) the environment, and (5) the elapsed time between measurements. Precision has two conditions: repeatability and reproducibility. Under repeatability conditions, factors such as (1) to (5) are considered to be constant and do not contribute to the variability of the measurement result. Under reproducibility conditions, the factors can vary. Repeatability and reproducibility are the two extremes of precision.

We assessed repeatability by means of the following methods: 
TABLE 1

\begin{tabular}{lc} 
Mean Value of the Optic Nerve Head Parameters \\
\hline Parameter & Mean \pm SD \\
\hline CDR & $0.51 \pm 0.14$ \\
\hline Disc area $\left(\mathrm{mm}^{2}\right)$ & $1.91 \pm 0.32$ \\
\hline Rim area $\left(\mathrm{mm}^{2}\right)$ & $1.32 \pm 0.21$ \\
\hline Cup volume $\left(\mathrm{mm}^{3}\right)$ & $0.14 \pm 0.11$ \\
\hline Horizontal CDR & $0.57 \pm 0.16$ \\
\hline Vertical CDR & $0.47 \pm 0.11$ \\
\hline $\begin{array}{l}\text { CDR }=\text { cup-to-disc area ratio; } S D=\text { standard deviation. } \\
{ }^{a} \text { All values represent the average of the three measurements. }\end{array}$
\end{tabular}

1. Repeated measurements analysis of variance: this was automatically calculated using Instat software 3.0 (GraphPad Software, San Diego, CA) for each of the ONH parameters.

2. Intraclass correlation coefficient (ICC): this is defined as the ratio of the between-subjects variance to the sum of the pooled within-subject variance and the between-subjects variance. It was automatically calculated using SPSS software 12.01 (SPSS Inc., Chicago, IL).

3. Coefficient of variation (COV): this was calculated as the within-subject SD (ie, the square root of the average of the within-subject variance) divided by the overall mean and was expressed as a percentage. ${ }^{22,23}$

4. Intrasession test-retest variability: this was calculated by multiplying the within-subject SD by $2.77 . .^{23}$

\section{RESULTS}

After excluding one subject due to poor quality of the scans (because errors resulted in the segmentation and 5.0 software does not have any editing capability), 32 eyes of 32 subjects (mean age: $56.4 \pm 19.2$ years) were analyzed.

Analysis of variance did not disclose any statistically significant difference among the three measurements for any parameter. The mean value of each parameter is reported in Table 1. Results for ICC, intrasession testretest variability, and COV are summarized in Table 2. For all parameters, the ICC was higher than 0.94 and the COV was lower than $4.5 \%$.

A comparison to previous studies about $\mathrm{ONH}$ repeatability of measurements obtained using Stratus OCT showed improved values in all cases.

\section{DISCUSSION}

Previous studies performed to investigateTD-OCT measurements have shown that topographic analysis of the $\mathrm{ONH}$ is of utmost importance for the assessment of the optic disc for many reasons. First, combining RNFL and ONH parameters results in the highest diagnostic accuracy for glaucoma. ${ }^{16}$ Second, $\mathrm{ONH}$ size influences the sensitivity of Stratus OCT with respect to glaucoma detection, because a decrease in sensitivity has been reported in subjects with larger discs. ${ }^{24} \mathrm{Third}$, $\mathrm{ONH}$ parameters play a major role in the clinical course of other diseases such as nonarteritic anterior ischemic optic neuropathy, Leber's hereditary optic neuropathy, and dominant optic atrophy. ${ }^{17,18,25}$ Fourth, optic disc size influences OCT RNFL thickness measurements in healthy eyes. ${ }^{26-28}$ Hence, the development of specific software for $\mathrm{ONH}$ analysis on Cirrus HD-OCT is a remarkable update for this instrument.

Repeatability of ONH parameter measurements by Cirrus HD-OCT has been recently assessed in a sample of glaucomatous eyes by Mwanza et al., who reported excellent results. ${ }^{29}$ Our study is the first to investigate repeatability of the same measurements in healthy eyes, an essential requisite to validate this software and develop future normative database for $\mathrm{ONH}$ parameters. Our data are in good agreement with those reported by Mwanza et al., although we found a slightly higher intravisit repeatability in most cases; for example, the COV of the disc area was $2.29 \%$ instead of $4.4 \%$ and the COV of the rim area was $2.38 \%$ instead of $6.6 \%$. This difference may be related to the presence of peripapillary atrophy in glaucomatous eyes and its absence in our sample, and the higher mean age in Mwanza et al.'s study (70.7 compared to 56.4 years in ours), with possible higher prevalence of cataract. Our data also suggest that Cirrus HD-OCT increases the repeatability of $\mathrm{ONH}$ parameter measurements compared to Stratus OCT, as is shown in Table 2. ${ }^{11,12}$ For example, disc area repeatability was higher than that reported by Kamppeter et al. (COV $=1.99 \%$ vs $5.7 \%$ for Cirrus HD-OCT and Stratus OCT, respectively), and the same applies for CDR (COV $=1.84 \%$ vs $15.3 \%$ for Cirrus HD-OCT and Stratus OCT, respectively). ${ }^{12}$

Our findings, together with those reported by 


\begin{tabular}{|c|c|c|c|c|c|c|}
\hline \multirow[b]{3}{*}{ Parameter } & \multicolumn{6}{|c|}{$\begin{array}{l}\text { TABLE } 2 \\
\text { ICC, Intrasession Test-Retest Variability, and COV of Each } \\
\text { Optic Nerve Head Parameter Measured by Cirrus HD-OCT }\end{array}$} \\
\hline & \multicolumn{2}{|c|}{ ICC (Lower 95\% Cl) } & \multicolumn{2}{|c|}{$\begin{array}{c}\text { Intrasession Test-Retest } \\
\text { Variability }\end{array}$} & \multicolumn{2}{|c|}{$\operatorname{cov}(\%)$} \\
\hline & $\begin{array}{l}\text { Current } \\
\text { Study }\end{array}$ & $\begin{array}{l}\text { Paunescu } \\
\text { et al. }{ }^{11}\end{array}$ & $\begin{array}{l}\text { Current } \\
\text { Study }\end{array}$ & $\begin{array}{l}\text { Paunescu } \\
\text { et al. }{ }^{11}\end{array}$ & $\begin{array}{l}\text { Current } \\
\text { Study }\end{array}$ & $\begin{array}{l}\text { Kamppetter } \\
\text { et al. }{ }^{12}\end{array}$ \\
\hline CDR & $0.99(0.99)$ & $0.97^{a}$ & 0.024 & $0.055^{b}$ & 1.68 & 15.3 \\
\hline Disc area $\left(\mathrm{mm}^{2}\right)$ & $0.98(0.96)$ & 0.66 & 0.121 & $0.637^{b}$ & 2.29 & 5.7 \\
\hline Rim area $\left(\mathrm{mm}^{2}\right)$ & $0.98(0.96)$ & $0.81^{a}$ & 0.087 & $0.332^{b}$ & 2.38 & 7.3 \\
\hline Cup volume $\left(\mathrm{mm}^{3}\right)$ & $0.99(0.99)$ & $0.84^{a}$ & 0.017 & $0.110^{\mathrm{b}}$ & 4.23 & - \\
\hline Horizontal CDR & $0.99(0.98)$ & $0.87^{\mathrm{a}}$ & 0.048 & $0.083^{b}$ & 3.03 & 8.5 \\
\hline Vertical CDR & $0.98(0.96)$ & $0.90^{\mathrm{a}}$ & 0.048 & $0.083^{b}$ & 3.65 & 8.4 \\
\hline \multicolumn{7}{|c|}{ 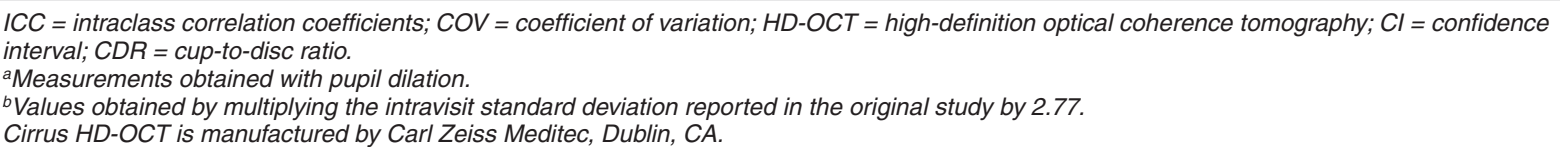 } \\
\hline
\end{tabular}

Mwanza et al.,29 reveal an additional advantage of SDOCT technology, which has already been reported to improve the repeatability and reproducibility of peripapillary RNFL thickness measurements. ${ }^{9,10,30}$ Although the most likely reason for the lower variability in Cirrus HD-OCT RNFL thickness measurements is the automatic positioning of the peripapillary circle around the $\mathrm{ONH}$, which reduces the changes in RNFL thickness related to manual location of the circle, ${ }^{30}$ the reason for the improved repeatability of $\mathrm{ONH}$ measurements is different and is likely to depend mostly on the higher number of scans used to reconstruct $\mathrm{ONH}$ topography. It should be remembered that Stratus OCT reconstructs $\mathrm{ONH}$ topography on the basis of six radial $\mathrm{B}$-scans in a spoke-like pattern centered on the $\mathrm{ONH}$; the OCT interpolates among the B-scans to provide measurements for the entire $\mathrm{ONH}$. In the case of Cirrus HD-OCT, interpolation is minimal because $\mathrm{ONH}$ topography is reconstructed on the basis of as many as $200 \mathrm{~B}$-scans (containing $200 \mathrm{~A}$-scans each). A second explanation for improved repeatability may be the higher transverse resolution of each B-scan: with Stratus OCT the best repeatability has been achieved with the Fast $\mathrm{ONH}$ acquisition protocol ${ }^{12}$ at $128 \mathrm{~A}$ scans, whereas the transverse resolution of Cirrus HD-OCT is $200 \mathrm{~A}$-scans. Given that the scans cover a 4- and 6-mm lateral field of view, respectively, the increased sampling density and better lateral resolution of the SD-OCT device are likely to support improved analyses. The axial resolution of Cirrus HD-OCT (5 $\mu \mathrm{m}$ ) is also higher than that of Stratus OCT (7 to 10 $\mu \mathrm{m})$. Increased resolution leads to better visualization of the Bruch's membrane opening (Figure).

A comparison of our results to those of a previous study assessing the repeatability of $\mathrm{ONH}$ measurements provided by another SD-OCT device (RTVue; Optovue Inc, Fremont, CA) show a better COV for Cirrus HD-OCT versus RTVue for most parameters, including CDR ratio (1.68\% vs $22.2 \%)$, cup volume (4.23 vs $29.1 \%$ ), horizontal CDR (3.03 vs $14.1 \%$ ), vertical CDR (3.65 vs $15.8 \%)$, and rim area $(2.38 \%$ vs $2.68 \%)$, and a slightly worse COV for disc area (2.29 vs $0.27 \%)$. The differences probably depend on the fact that the RTVue uses a different method to obtain measurements of the $\mathrm{ONH}$, whose disc margin is drawn manually on the en-face image for each scan; in addition, other factors, such as differences in age, refractive error, and inclusion of eyes with peripapillary atrophy (which was an exclusion criterion in our sample) might have played a role. ${ }^{10}$

Further studies are needed to determine whether the higher repeatability of $\mathrm{ONH}$ measurements can improve the diagnostic performance of SD-OCT. Recent articles evaluating RNFL thickness measurements have shown that the diagnostic sensitivity and specificity of Cirrus HD-OCT are similar to those of TD-OCT. ${ }^{30-33}$ Similar results have been reported with another SDOCT device. ${ }^{34}$ Only one study found better values with 
SD-OCT than TD-OCT. ${ }^{35}$ Given that previous studies with Stratus OCT have reported increased sensitivity in detecting glaucoma after combining data from RNFL thickness and ONH measurements, ${ }^{16}$ it is likely that combining the same data will enhance the diagnostic performance of Cirrus HD-OCT. Meanwhile, it has already been shown that there is no difference in the ability of ONH parameters and RNFL thickness measurements, as measured with Cirrus HD-OCT, to distinguish between healthy and glaucomatous eyes. ${ }^{36}$

The current study has some limitations. We did not include eyes with papilledema, optic disc drusen, optic atrophy (due to glaucoma or other causes), or peripapillary atrophy; hence our findings cannot be directly applied to these cases (a prospective study evaluating eyes with Leber's hereditary optic neuropathy, however, is being performed). Nor did we evaluate intersession reproducibility. These issues warrant further investigation.

We observed that $\mathrm{ONH}$ parameters measured by Cirrus HD-OCT show excellent repeatability in healthy eyes, with less variability compared to TDOCT. Further studies are needed to detect the benefits resulting from this finding.

\section{REFERENCES}

1. Huang D, Swanson EA, Lin CP, et al. Optical coherence tomography. Science. 1991;254:1178-1181.

2. Knight OJ, Chang TR, Feuer WJ, Budenz DL. Comparison of retinal nerve fiber layer measurements using time domain and spectral domain optical coherence tomography. Ophthalmology. 2009;116:1271-1277.

3. Wojtkowski M, Leitgeb R, Kowalczyk A, Bajraszewski T, Fercher AF. In vivo human retinal imaging by Fourier domain optical coherence tomography. J Biomed Opt. 2002;7:457-463.

4. Lin SC, Singh K, Jampel HD, et al. Optic nerve head and retinal nerve fiber layer analysis: a report by the American Academy of Ophthalmology. Ophthalmology. 2007;114:1937-1949.

5. Lee EJ, Kim TW, Park KH, Seong M, Kim H, Kim DM. Ability of Stratus OCT to detect progressive retinal nerve fiber layer atrophy in glaucoma. Invest Ophthalmol Vis Sci. 2009;50:662-668.

6. Savini G, Bellusci C, Carbonelli M, et al. Detection and quantification of retinal nerve fiber layer thickness in optic disc edema using Stratus OCT. Arch Ophthalmol. 2006;124:1111-1117.

7. Barboni P, Savini G, Valentino ML, et al. Retinal nerve fiber layer evaluation by optical coherence tomography in Leber's hereditary optic neuropathy. Ophthalmology. 2005;112:120-126.

8. Savini G, Barboni P, Valentino ML, et al. Retinal nerve fiber layer evaluation by optical coherence tomography in unaffected carriers with Leber's hereditary optic neuropathy mutations. Ophthalmology. 2005;112:127-131.

9. Vizzeri G, Weinreb RN, Gonzáles-García AO, et al. Agreement between spectral-domain and time-domain OCT for measuring RNFL thickness. Br J Ophthalmol. 2009;93:775-781.

10. Gonzáles-García AO, Vizzeri G, Bowd C, Medeiros FA, Zangwill LM, Weinreb RN. Reproducibility of RTVue retinal nerve fiber layer thickness and optic disc measurements and agreement with Stratus optical coherence tomography measurements. Am J Ophthalmol.
2009; 147:1067-1074

11. Paunescu LA, Schuman JS, Price LL, et al. Reproducibility of nerve fiber layer thickness, macular thickness, and optic nerve head measurements using StratusOCT. Invest Ophthalmol Vis Sci. 2004;45:1716-1724.

12. Kamppeter BA, Schubert KV, Budde WM, Degenring RF, Jonas JB. Optical coherence tomography of the optic nerve head: interindividual reproducibility. J Glaucoma. 2006;15:248-254.

13. Huang ML, Chen HY, Lin JC. Rule extraction for glaucoma detection with summary data from StratusOCT. Invest Ophthalmol Vis Sci. 2007:48:244-250

14. Manassakorn A, Nouri-Mahdavi K, Caprioli J. Comparison of retinal nerve fiber layer thickness and optic disk algorithms with optical coherence tomography to detect glaucoma. Am J Ophthalmol. 2006;141:105-115.

15. Naithani P, Sihota R, Sony P, et al. Evaluation of optical coherence tomography and Heidelberg retinal tomography parameters in detecting early and moderate glaucoma. Invest Ophthalmol Vis Sci. 2007;48:3138-3145.

16. Medeiros FA, Zangwill LM, Bowd C, Vessani RM, Susanna R Jr, Weinreb RN. Evaluation of retinal nerve fiber layer, optic nerve head, and macular thickness measurements for glaucoma detection using optical coherence tomography. Am J Ophthalmol. 2005;139:44-55.

17. Ramos Cdo V, Bellusci C, Savini G, et al. Association of optic disc size with development and prognosis of Leber's hereditary optic neuropathy. Invest Ophthalmol Vis Sci. 2009;50:1666-1674.

18. Barboni P, Carbonelli M, Savini G, et al. OPA1 mutations associated with dominant optic atrophy influence optic nerve head size. $O p h$ thalmology. 2010;117:1547-1553.

19. Strouthidis NG, Yang H, Reynaud JF, et al. Comparison of clinical and spectral domain optical coherence tomography optic disc margin anatomy. Invest Ophthalmol Vis Sci. 2009;50:4709-4718.

20. Strouthidis NG, Yang H, Fortune B, Downs JC, Burgoyne CF. Detection of optic nerve head neural canal opening within histomorphometric and spectral domain optical coherence tomography data sets. Invest Ophthalmol Vis Sci. 2009;50:214-223.

21. British Standards Institution, BS ISO 5725-1. Accuracy (Trueness and Precision) of Measurement Methods and Results_Part 1: General Principles and Definitions. London: BSI; 1994:1-26.

22. Budenz DL, Fredette MJ, Feuer WJ, Anderson DR. Reproducibility of peripapillary retinal nerve fiber thickness measurements with Stratus OCT in glaucomatous eyes. Ophthalmology. 2008;115:661-666.

23. Bland JM, Altman DG. Measurement error. BMJ. 1996;313:744-746.

24. Medeiros FA, Zangwill LM, Bowd C, Sample PA, Weinreb RN. Influence of disease severity and optic disc size on the diagnostic performance of imaging instruments in glaucoma. Invest Ophthalmol Vis Sci. 2006; $47: 1008-1015$.

25. Chan CK, Cheng AC, Leung CK, et al. Quantitative assessment of optic nerve head morphology and retinal nerve fibre layer in nonarteritic anterior ischaemic optic neuropathy with optical coherence tomography and confocal scanning laser ophthalmoloscopy. $\mathrm{BrJOph}$ thalmol. 2009;93:731-735.

26. Savini G, Zanini M, Carelli V, Sadun AA, Ross-Cisneros FN, Barboni P. Correlation between retinal nerve fiber layer thickness and optic nerve head size: an optical coherence tomography study. BrJ Ophthalmol. 2005;89:489-492.

27. Savini G, Barboni P, Carbonelli M, Zanini M. The effect of scan diameter on retinal nerve fiber layer thickness measurement using Stratus optical coherence tomography. Arch Ophthalmol. 2007;125:901-905.

28. Budenz DL, Anderson DR, Varma R, et al. Determinants of normal retinal nerve fiber layer thickness measured by StratusOCT. Ophthalmology. 2007;114:1046-1052.

29. Mwanza JC, Chang RT, Budenz DL, et al. Reproducibility of peripapillary retinal nerve fiber layer thickness and optic nerve head parameters measured with Cirrus HD-OCT in glaucomatous eyes. Invest Ophthalmol Vis Sci. 2010;51:5724-5730.

30. Leung CK, Cheung CY, Weinreb RN, et al. Retinal nerve fiber layer imaging with spectral-domain optical coherence: a variability and diagnostic performance study. Ophthalmology. 2009;116:1257-1263.

31. Chang RT, Knight OJ, Feuer WJ, Budenz DL. Sensitivity and specificity of time-domain versus spectral-domain optical coherence to- 
mography in diagnosing early to moderate glaucoma. Ophthalmology. 2009;116:2294-2299.

32. Moreno-Montañes J, Olmo N, Alvarez A, García N, Zarranz-Ventura J. Cirrus high-definition optical coherence tomography compared to Stratus optical coherence tomography in glaucoma diagnosis. Invest Ophthalmol Vis Sci. 2010;51:335-343.

33. Jeoung JW, Park KH. Comparison of Cirrus OCT and Stratus OCT on the ability to detect localized retinal nerve fiber layer defects in preperimetric glaucoma. Invest Ophthalmol Vis Sci. 2010;51:938-945.

34. Sehi M, Grewal DS, Sheets CW, Greenfield DS. Diagnostic ability of Fourier-domain vs time-domain optical coherence tomography for glaucoma detection. Am J Ophthalmol. 2009;148:597-605.

35. Sung KR, Kim DY, Park SB, Kook MS. Comparison of retinal nerve fiber layer thickness measured by Cirrus HD and Stratus optical coherence tomography. Ophthalmology. 2009;116:1264-1270.

36. Mwanza JC, Oakley J, Budenz DL, Anderson DR; Cirrus Optical Coherence Tomography Normative Database Study Group. Ability of Cirrus HD-OCT optic nerve head parameters to discriminate normal from glaucomatous eyes. Ophthalmology. 2010;118:241-248. 
Reproduced with permission of the copyright owner. Further reproduction prohibited without permission. 